\title{
Identification of circRNAs involved in the development of hepatocellular carcinoma after insufficient radiofrequency ablation
}

\author{
Jun-Wei CHEN ${ }^{1, *}$, Li-Sha LAI ${ }^{2, *}$, Jun-Yang LUO ${ }^{1}$, Chu-Ren ZHOU ${ }^{1}$, Min-Gan LI' ${ }^{1}$, Ming-Sheng HUANG ${ }^{1, *}$, Ping WU ${ }^{3, *}$ \\ ${ }^{1}$ Department of Interventional Radiology, The Third Affiliated Hospital of Sun Yat-sen University, Guangzhou, Guangdong, China; ${ }^{2}$ Department \\ of Radiology, Guangzhou First People's Hospital, School of Medicine, South China University of Technology, Guangzhou, Guangdong, China; \\ ${ }^{3}$ Department of Gastroenterology, Maoming People's Hospital, Southern Medical University, Maoming, Guangdong, China
}

*Correspondence: huangmsh@mail.sysu.edu.cn; wujunda1208@126.com

${ }^{*}$ Contributed equally to this work.

Received August 17, 2021 / Accepted December 29, 2021

\begin{abstract}
Previous studies have reported that circular RNAs (circRNAs) play a key role in the pathogenesis and progression of various diseases. In the present study, we aimed to identify potential circRNAs associated with the progression of hepatocellular carcinoma (HCC) after insufficient radiofrequency ablation (IRFA). A xenograft mouse IRFA model was initially established, and immunohistochemical staining (IHC) and polymerase chain reaction (PCR) were performed to confirm the expression of programmed cell death-ligand 1 (PD-L1) and vascular endothelial growth factor receptor-1 (VEGFR-1). CircRNA expression alterations were screened by next-generation sequencing (RNA-seq). Gene ontology (GO) and Kyoto Encyclopedia of Genes and Genomes (KEGG) analyses were conducted to predict the function of genes coding differentially expressed circRNAs. The selected circRNAs were validated utilizing PCR and Sanger sequencing. The relationships between circRNAs, microRNAs, PD-L1, and VEGFR-1 were predicted by bioinformatics. Overall, a total of 612 circRNAs were differentially expressed in IRFA-treated subcutaneous tumorigenesis tissue. Among them, 435 circRNAs were significantly upregulated and 177 circRNAs were downregulated. GO and KEGG analyses were employed to predict the functions of these circRNAs. Thereafter, quantitative reverse transcription PCR (qRT-PCR) assays determined that these seven circRNAs were overexpressed in the IRFA group, which was consistent with the RNA-seq results. Based on the bioinformatic analysis, seven circRNAs confirmed by Sanger sequencing were predicted to likely regulate PD-L1 and VEGFR-1 expression levels by functioning as sponges for microRNAs (miRNAs) and forming a circRNA-miRNA-PD-L1/VEGFR-1 regulatory network. Finally, IHC and qRT-PCR of PD-L1 and VEGFR-1 confirmed the activation of this pathway. Taken together, we report that differentially expressed circRNAs might simultaneously regulate PD-L1 and VEGFR-1 in the IRFA tissues, which provides a novel view of circRNAs in HCC progression after the IRFA procedure.
\end{abstract}

Key words: hepatocellular carcinoma; insufficient radiofrequency ablation; circRNAs; PD-L1; VEGFR-1

Hepatocellular carcinoma (HCC) is the fifth most common malignancy and the third leading cause of cancerrelated deaths worldwide [1,2]. Radiofrequency ablation (RFA) is an effective local treatment for curative procedures in patients with cirrhosis of the liver and HCC with small nodules [3]. However, insufficient RFA (IRFA) treatment for $\mathrm{HCC}$ has been reported as a risk factor for early recurrence, especially with large tumor lesions. In addition, some studies have found that IRFA might enhance local tumor angiogenesis and promote rapid progression of residual $\operatorname{HCC}[4,5]$, but the precise molecular mechanism remains elusive.

Circular RNAs (circRNAs) are a new class of endogenous noncoding RNAs produced by the noncanonical mode of
RNA splicing that features a covalently closed loop with no 5'-3' polarity [6]. Previous studies have revealed that circRNAs broadly participate in the initiation and development of various diseases, especially in malignant tumors, including esophageal squamous cell carcinoma (ciRS-7, circITCH) [7, 8], gastric cancer (circPVT1, circLARP4) [9, 10], colorectal cancer (circITGA7, circCCDC66) [11, 12], and HCC (circMTO1, cSMARCA5) [13, 14]. Mechanistically, it has been suggested that many circRNAs can serve as efficient miRNA sponges, which bind miRNAs through imperfect nucleic base pairing to regulate gene expression [15-17]. The dysregulation and functions of miRNAs have been extensively investigated in almost every biological process 
[18]. However, the expression profiles and functions of the newly identified circRNAs in specific biological activities still require further exploration.

In our present study, to explore the circRNA profile and the potential functions of circRNAs in IRFA-treated HCC, we established a mouse IRFA model of HCC to identify differentially altered circRNAs through next-generation sequencing. Subsequently, bioinformatics analysis was performed to determine the potential mechanism of these circRNAs. Taken together, we provide novel data to help improve our diagnostic capabilities and knowledge of the progression of HCC with IRFA treatment.

\section{Materials and methods}

Xenograft mouse IRFA model establishment. 10 female $\mathrm{BALB} / \mathrm{c}$ nude mice (4 weeks old, 18-20 g) were purchased from Guangdong Medical Laboratory Animal Center (Guangdong, China) and housed under specific pathogenfree conditions. Two hundred microliters of LM3 cell suspension $\left(2 \times 10^{6}\right)$ were injected into nude mice subcutaneously. Tumor formation was observed every day, and when the tumor diameter reached $12-15 \mathrm{~mm}$, the RFA procedure was performed. These 10 nude mice were randomly divided into two groups: the IRFA groups were as follows: RFA was performed with a lower energy protocol, in which the outer power was $5 \mathrm{~W}$ and the duration was $30 \mathrm{~s}$. This ensured the presence of residual cancer. Mice in the control group (sham group) were sham-operated by inserting a needle electrode into the tumor without performing ablation. This study was approved by the Ethical Committee of the Third Affiliated Hospital of Sun Yat-sen University.

$\mathrm{H} \& \mathrm{E}$ staining and immunohistochemical (IHC) staining of PD-L1 and VEGFR-1 in IRFA HCC tissues. IRFA group and sham group HCC tissues were fixed with $10 \%$ neutral buffered formalin and embedded in paraffin. Tissue sections were processed and stained with $\mathrm{H} \& \mathrm{E}$ as described previously. To conduct IHC, tissue sections were deparaffinized with xylene, rehydrated with various grades of ethanol $(100 \%, 95 \%$, and $70 \%)$, antigen unmasked with the heating-induced antigen repair technique citric acid buffer ( $\mathrm{pH} 6.0,95^{\circ} \mathrm{C}$ ), and blocked with 5\% BSA. Subsequently, the sections were incubated in succession with primary antibodies PD-L1 (BM4816, 1:20, Wuhan Boster Biological Technology, LTD, China), VEGFR-1 (ab32152, 1:100, Abcam, UK), CD31 (11265-1-ap, 1:500, Proteintech, USA), secondary antibody HRP-conjugated secondary mouse anti-rabbit IgG antigen (Wuhan Boster Biological Technology, LTD, China), and DAB ( $50 \mu \mathrm{l}$ DAB stock solution was mixed with $50 \mu \mathrm{l}$ hydrogen peroxide solution in $1 \mathrm{ml}$ PBS) substrate at the optimized concentration for color development. A TUNEL assay was performed with the DeadEnd $^{\mathrm{Tm}}$ Fluorometric TUNEL System (G3250, Promega, USA) to detect tumor apoptosis according to the manufacturer's instructions.
CircRNA extraction and quality control. Total RNA from both groups was extracted by using TRIzol reagent (Invitrogen, USA) according to the manufacturer's instructions. DNase I (Takara, Dalian, China) was used to digest DNA, RiboMinus ${ }^{\mathrm{Tm}}$ Eukaryote Probe Mix v2 was used to remove the rRNA, and RNase R (RNR07250, Epicentre, USA) was used to digest the linear RNA. RNA concentration was measured with a NanoDrop ND-1000 (Thermo Fisher Scientific, Waltham, MA, USA). Only RNA samples with suitable RNA electrophoresis results (28S/18S: 1.6-2.0) and RNA integrity number (RIN) $\geq 6$ were analyzed further. cDNA was synthesized using PrimeScript ${ }^{\mathrm{Tm}}$ RT Master Mix (TaKaRa, Dalian, China) according to the manufacturer's protocol (with random primers).

Identification and quantification of circRNAs. Clean reads were obtained from the raw data using an Illumina HiSeqTM 2000 (Illumina, USA). The differentially expressed circRNAs were selected using the following filter criteria: $\log 2$ Ratio (IRFA/Sham) $>1$, and $\mathrm{p}<0.05$. Gene ontology $(\mathrm{GO})$ and Kyoto Encyclopedia of Genes and Genomes (KEGG) analyses were performed to evaluate the function of the differentially expressed circRNAs between the IRFA group and sham group.

Quantitative real-time polymerase chain reaction (qRT-PCR) analysis. Based on the circRNA interaction network in which circRNAs can simultaneously regulate PD-L1 and VEGFR-1 (circRNA-miRNA-PD-L1/ VEGFR-1) in the IRFA group, circRNAs with more than 1 binding site (circRNA combined with miRNA) and lengths between 500 and 3,000 bp were selected for further qRT-PCR analysis. Meanwhile, qRT-PCR were performed to detect the expression of PD-L1 and VEGFR-1. qRT-PCR was performed with ChamQ SYBR qRT-PCR Master Mix (Q341-03, Vazyme, China) conducted with Roche Applied Science Light Cycler 480II Real-time PCR systems (Roche Applied Science, Indianapolis, IN, USA) in accordance with the manufacturer's instructions. A comparative cycle threshold (Ct) method was used to analyze the gene expression level, and all results are expressed as the mean \pm standard deviation (SD) from different circRNAs. The primers used for qRT-PCR are listed in Supplementary Table S1. All qRT-PCR procedures were performed at least three times.

Agarose gel electrophoresis and Sanger sequencing. To verify the circular structure of the circRNA, we designed a pair of convergent and divergent primers and verified their head-to-tail splicing using PCR and Sanger sequencing. RT-PCR products were inserted into a T vector for Sanger sequencing by determining their full length by Sangon Biotech (Shanghai, People's Republic of China).

Statistical analysis. All statistical data were analyzed using SPSS 13.0 (SPSS Inc., USA). All data are presented as the mean \pm SD. CircRNAs demonstrating log2Ratio (IRFA/ Sham) $>1$ and $p<0.05$ were regarded as significantly differentially expressed. Statistical significance of the levels of 
circRNAs was tested by Student's t-test, and a $\mathrm{p}<0.05$ was considered statistically significant.

\section{Results}

IRFA potentiated proliferation of HCC cells in vivo. To assess whether IRFA treatment affects tumor growth in vivo, we successfully established an IRFA model by subcutaneously transfecting LM3 cells into nude mice. As shown in Figure 1A, the HCC tumors in the IRFA group grew faster than those in the sham group. Tumor volume enlargement and larger average tumor weight were observed in the IRFA group compared with the control group (Figures 1B, 1C). These results indicated that IRFA treatment exerted stronger biological malignancy with the enhanced proliferative ability of HCC.

Expression profiles of circRNAs in IRFA HCC tissues. Given that circRNAs play key roles in the development and progression of various tumors $[6,15]$, we hypothesized that circRNAs might be implicated in the malignant process of IRFA HCC. Therefore, circRNA sequencing was conducted, and most circRNAs were produced from protein-coding exons, some were from introns, and others were intergenic. Differentially expressed circRNAs are displayed by a heatmap after fold-change filtering in Figure 2A. As a result, 612 circRNAs were found to be differentially expressed, of which 435 circRNAs were upregulated and 177 circRNAs were downregulated by more than two-fold in IRFA HCC compared to controls. Among these, a majority of differentially expressed circRNAs ranged in length from $250 \mathrm{bp}$ to 700 bp (Figure 2B). Moreover, many overexpressed circRNAs mainly originated from chromosomes 2,3 , and 8 , while the suppressed circRNAs were mainly generated from chromosomes 1, 2, and 7 (Figure 2C). In general, the expression profiles of circRNAs were changed dramatically in HCC after IRFA treatment. These data suggested that alterations in circRNA expression were associated with the IRFA model.

GO and pathway analyses of the parental genes of differentially expressed circRNAs. Considering that abnormal circRNA expression could lead to alterations in gene products that may contribute to tumor biology, including cell proliferation, apoptosis, angiogenesis, and metastasis [19-21], and to gain more insight into the biological functions of these differentially expressed circRNAs in IRFA HCC, the parental genes encoding differentially regulated circRNAs were further analyzed using DAVID. GO analyses were performed on three different categories, namely, BP, CC and MF, and these enrichments GO entries were shown in Figure 3A. Moreover, a KEGG pathway analysis was performed, and the pathways are listed in Figure 3B. Results showed that the most enriched and meaningful pathways were related to metabolic pathways, viral carcinogenesis, the Ras signaling pathway, the Wnt signaling pathway, the cAMP signaling pathway, and focal adhesion. These analyses indicated that these parental genes encoding differentially regulated circRNAs partici- pated in aggressive biological processes of HCC cells in the IRFA group.

The preliminary validation of circRNAs array. To authenticate the reliability of the circRNA array results, seven upregulated circRNAs in the IRFA group (hsa_circ_0085323, hsa_circ_0009102, hsa_circ_0070033, hsa_circ_0002617, hsa_circ_0002642, hsa_circ_0002048, hsa_circ_0003045) were selected for confirmation by qRT-PCR. The results were consistent with the sequencing data, demonstrating that this circRNA profile is highly reliable, as shown in Figures 4A-4G. Additionally, divergent primer pairs for the seven differentially changed circRNAs were designed in the IRFA group. The specificity of the products was confirmed

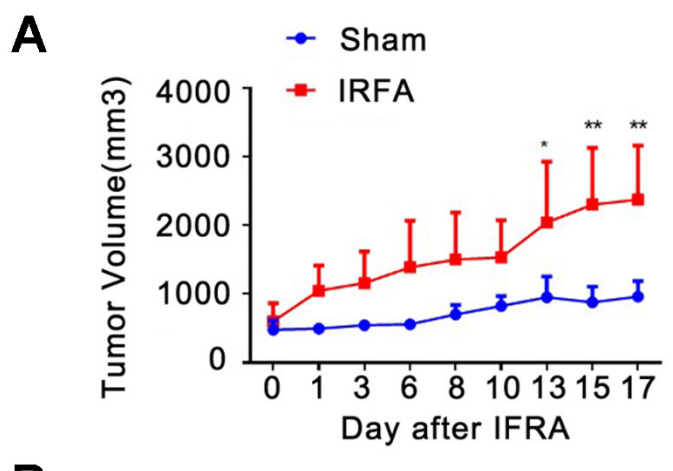

B
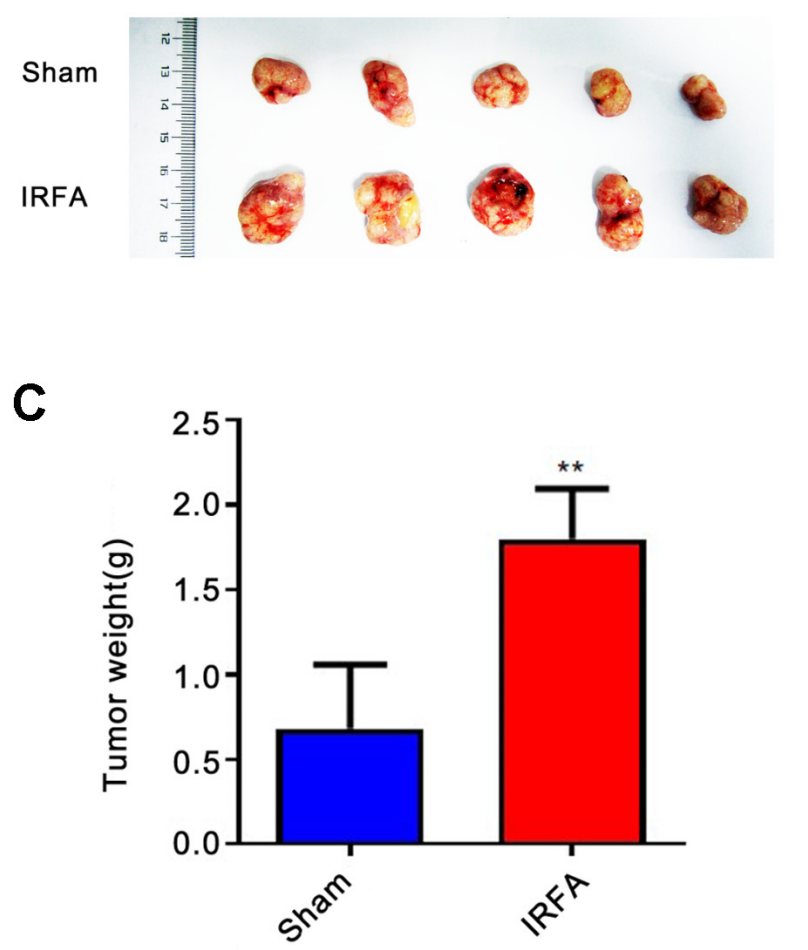

Figure 1. IRFA promoted tumor growth in vivo. A) IRFA-treated HCC cells potentiated tumor growth. B) Representation image of xenograft tumor in nude mice $(n=5)$. C) Analysis of tumor weight after xenograft tumor was removed. 


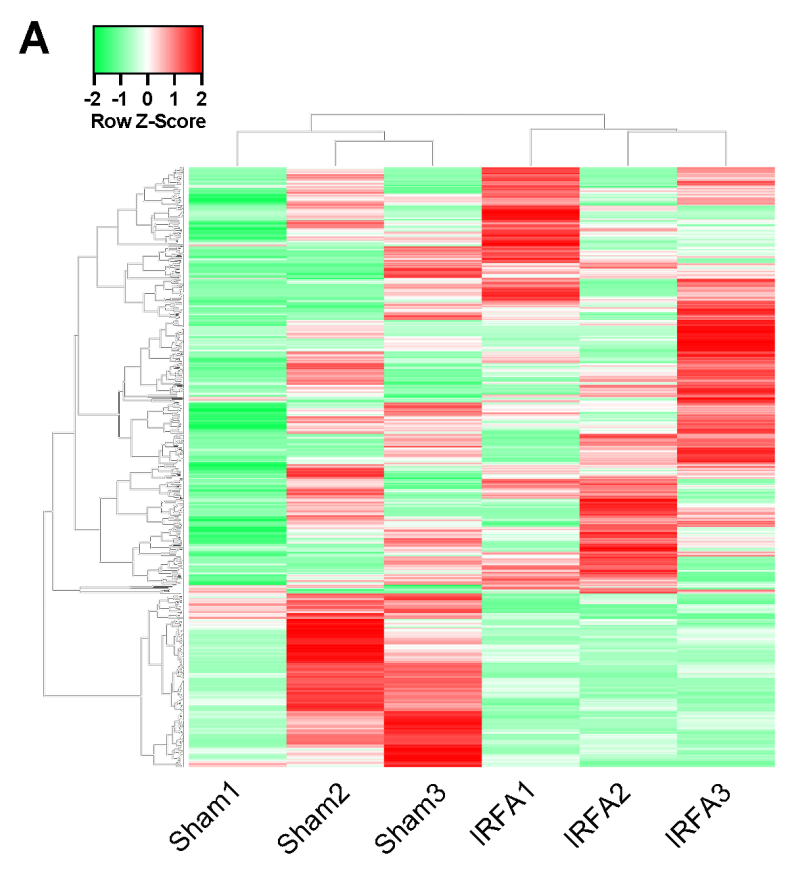

C

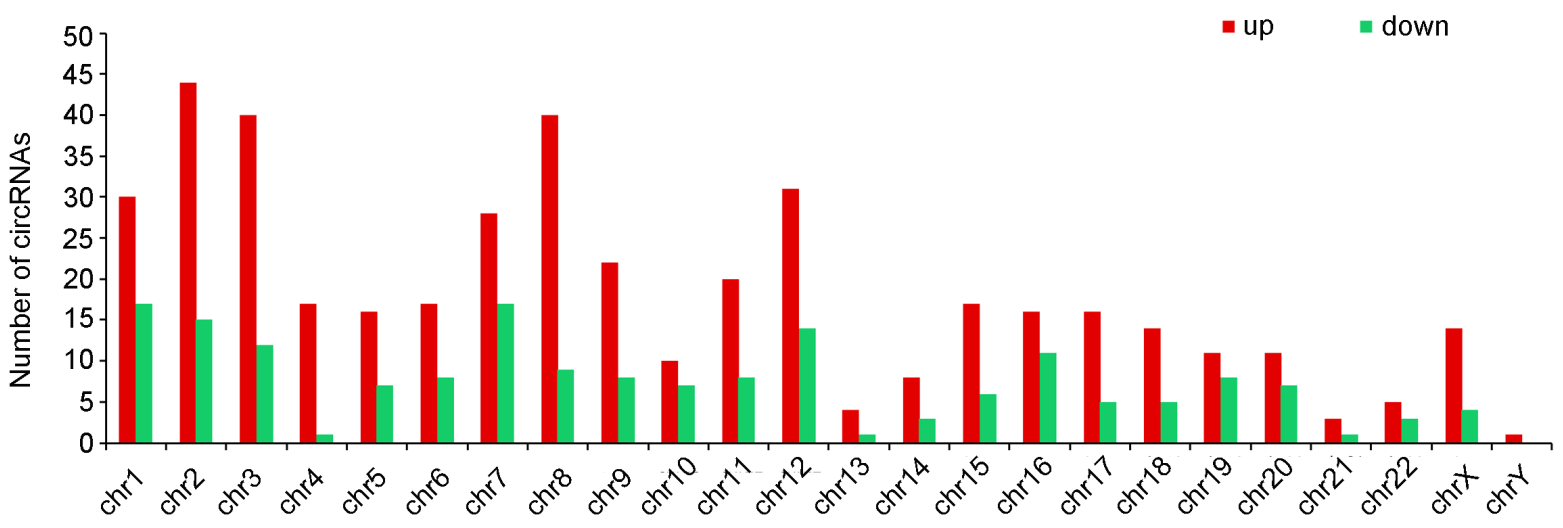

B

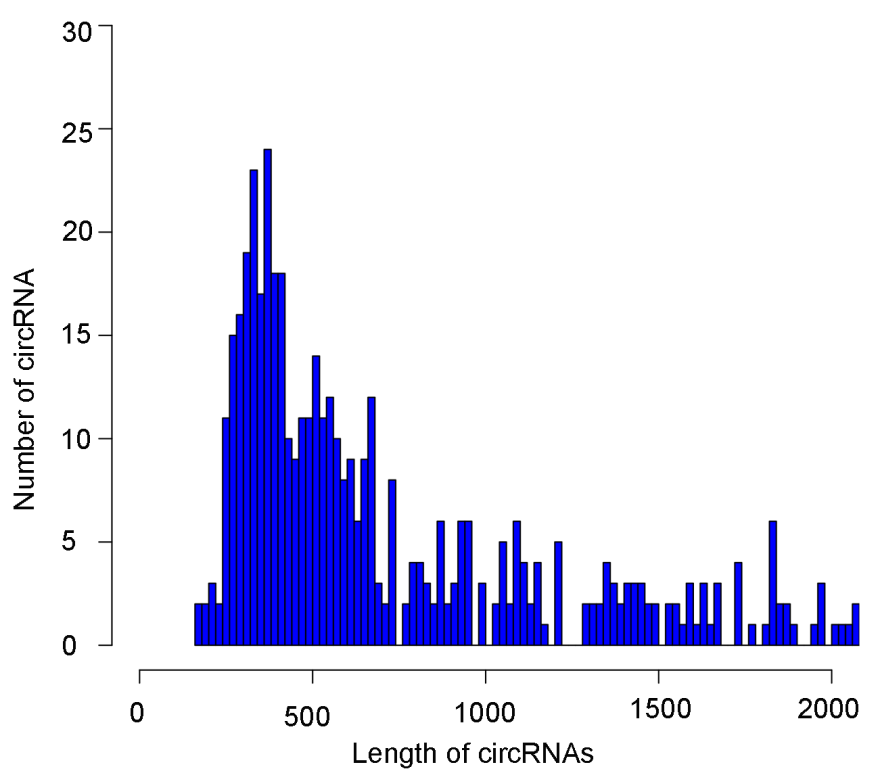

Figure 2. Expression profiles of circRNAs in IRFA model tumor tissues. A) Heatmap of differentially expressed circRNAs. B) The length distributions of differentially expressed circRNAs. C) Genomic distributions of differentially expressed circRNA.

using electrophoresis, and the splicing-site sequence of the seven circRNAs was confirmed by Sanger sequencing (Figures 5A-5G).

The circRNA/miRNA/PD-L1 and VEGFR-1 axes might promote the development of HCC after IRFA treatment. Previous studies have suggested that circRNAs mainly act as miRNA sponges to bind target miRNAs and then regulate gene expression [17]. It has been reported that VEGFR-1 and PD-L1 play a tumor-promoting role in HCC tissues. To investigate whether these seven differentially altered circRNAs had the potential to affect the mRNA expression levels of target genes by serving as "miRNA sponges" in IRFA HCC, bioinformatic analyses were performed using miRanda datasets to predict the circRNAs interacting with miRNAs. Results demonstrated that these seven circRNAs might target the 


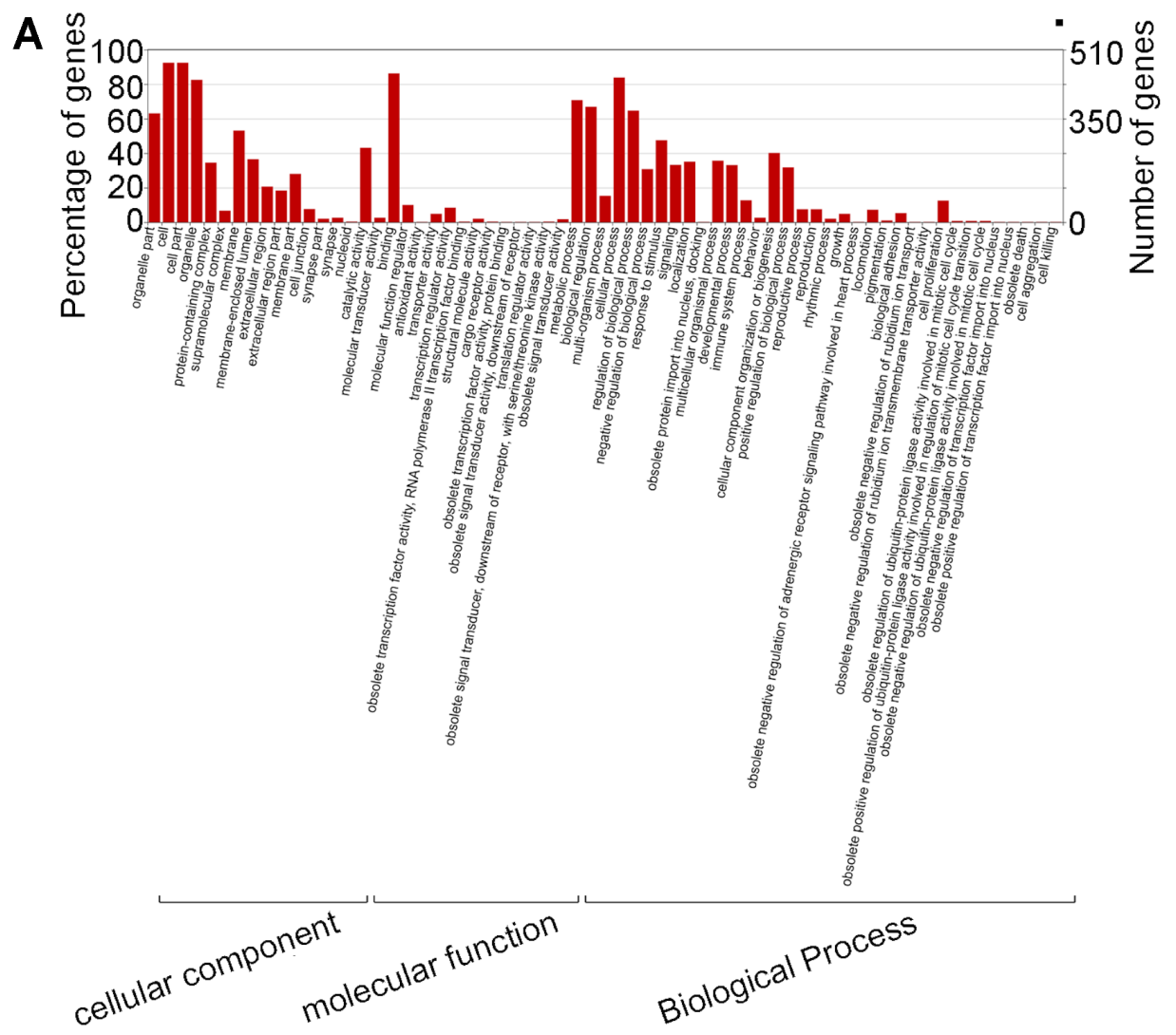

B

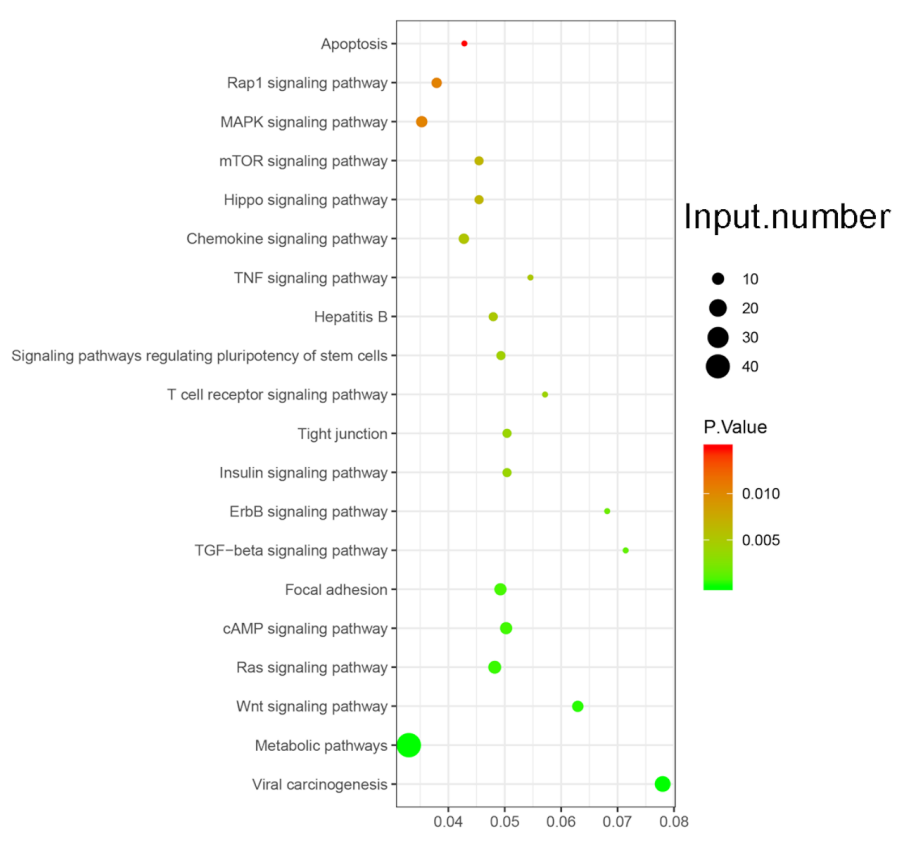

Pathway RichFactor

Figure 3. Expression profiles of circRNAs in IRFA model tumor tissues. A) circRNA-encoding genes were categorized by GO subcellular components, molecular function, and biological processes separately. B) KEGG signaling pathways with significant enrichment of genes encoding circRNAs differentially expressed in the IRFA group. 
A

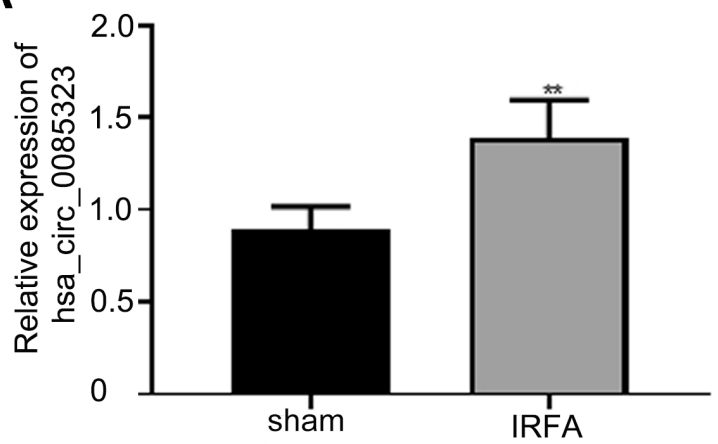

C

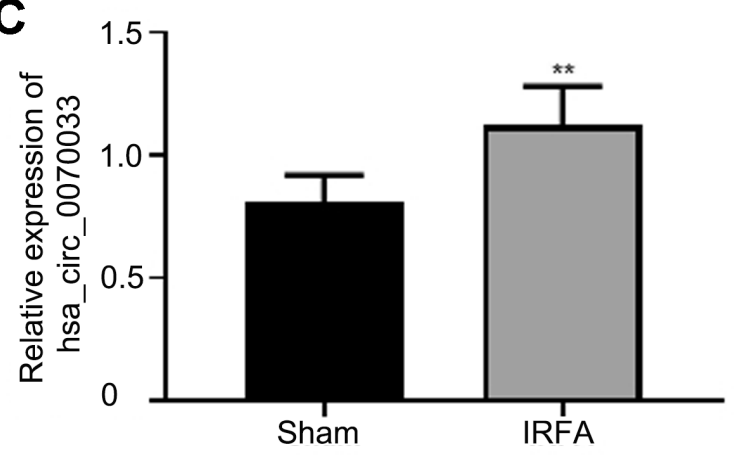

E

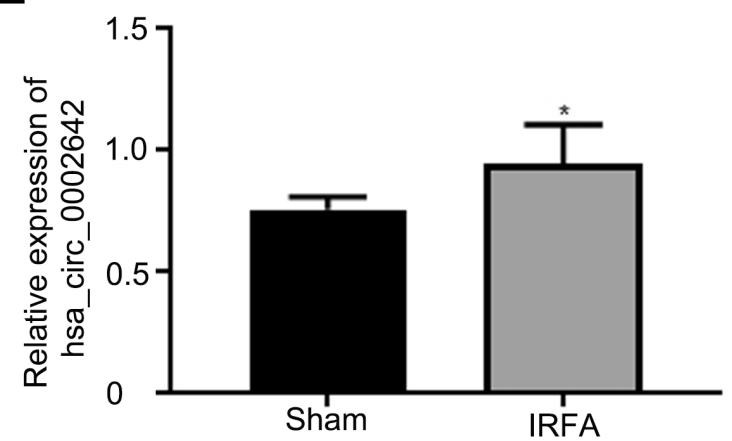

G

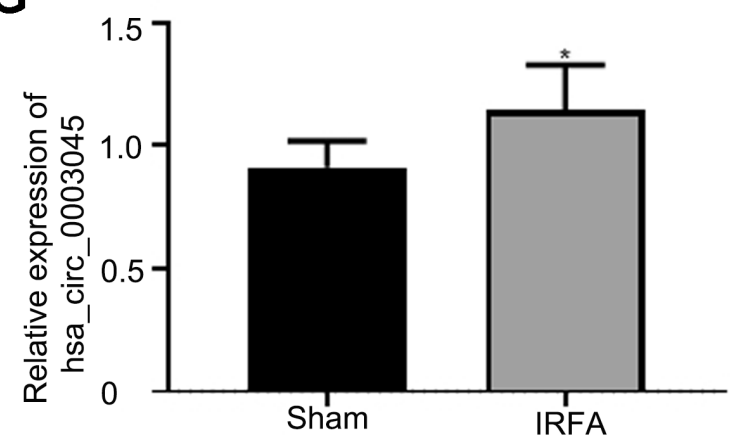

B

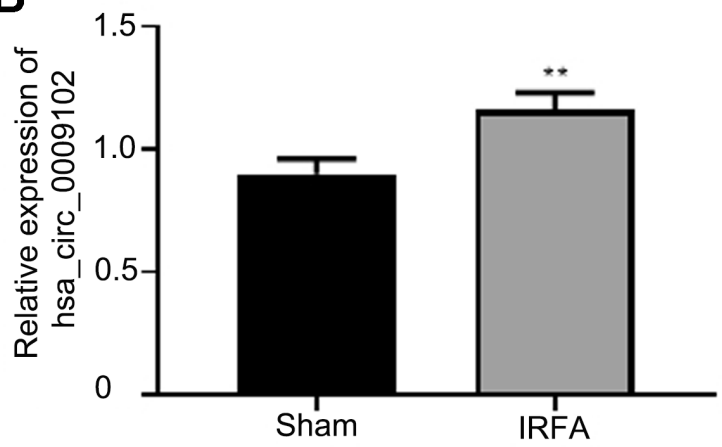

D

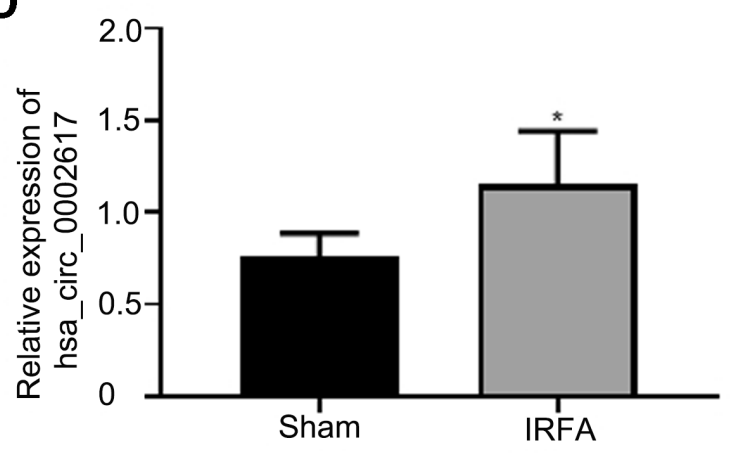

F

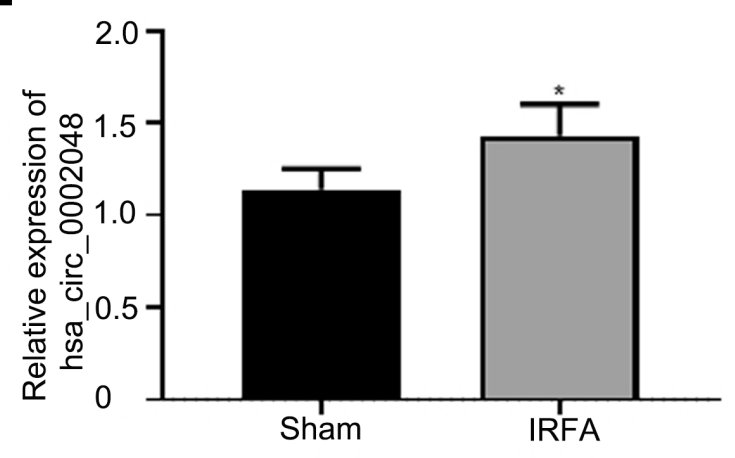

Figure 4. The expressions of seven circRNAs were determined in the IRFA group and control group. A-G) The relative seven circRNAs abundance detected by $\mathrm{qRT}$-PCR in IRFA-treated tumor tissues and the control group. Data were expressed as the mean \pm SD and reproduced in three independent experiments. ${ }^{\star} \mathrm{p}<0.05,{ }^{\star *} \mathrm{p}<0.01,{ }^{\star * *} \mathrm{p}<0.001$ 
A
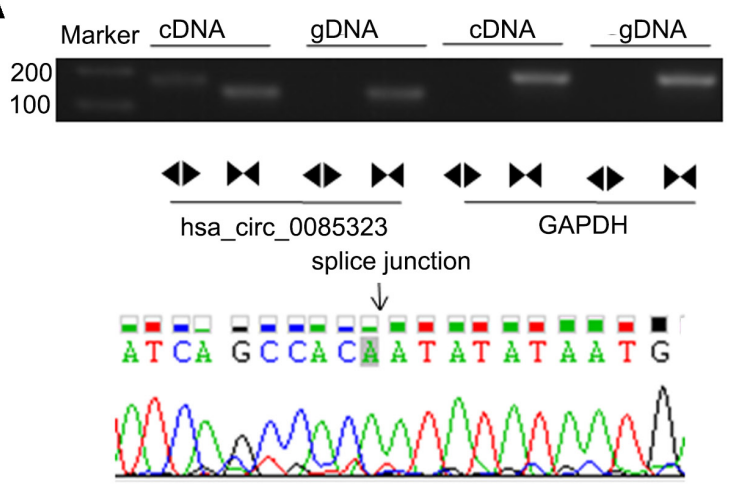

C
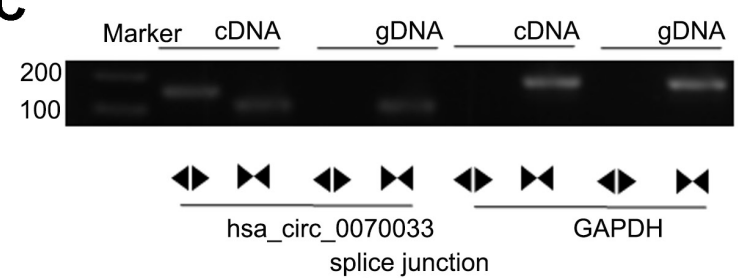

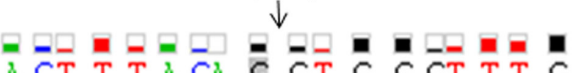

A CT T T A CA G GT G G GT T T G

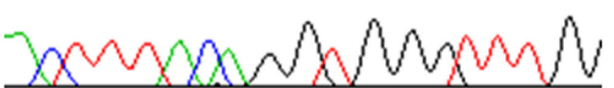

E

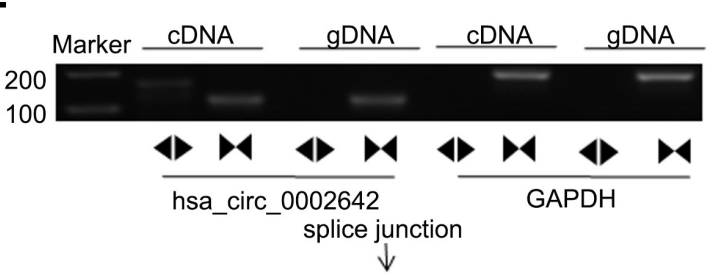

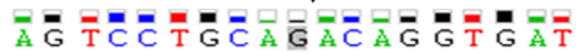

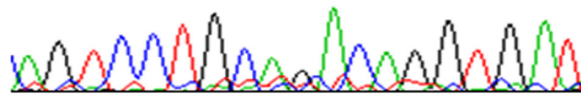

G

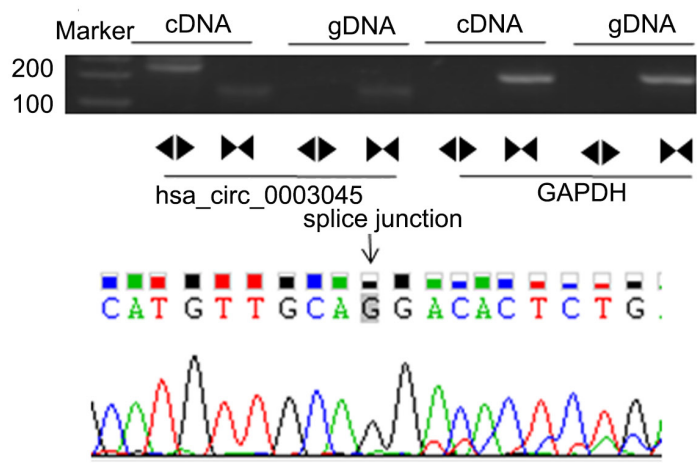

B Marker cDNA gDNA cDNA gDNA

200 100

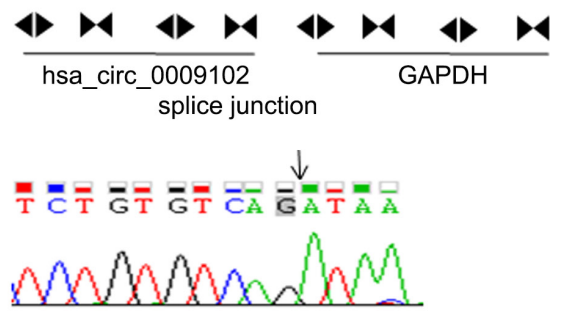

D Marker CDNA gDNA CDNA gDNA

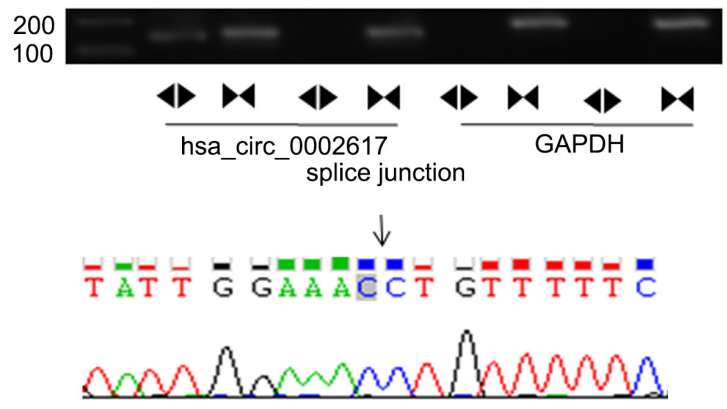

$\mathbf{F}$

Marker CDNA gDNA CDNA gDNA

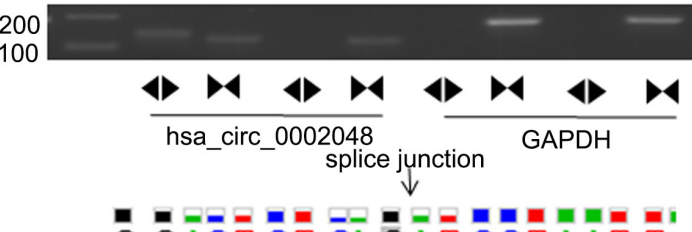

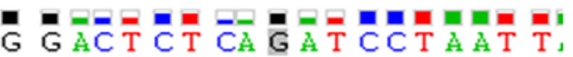

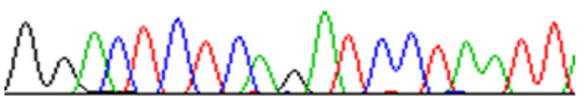

Figure 5. Validation of seven differentially circRNAs. A-G) circRNAs were verified by RT-PCR and amplified by divergent in cDNA but not in genomic DNA (gDNA). The sequence of back-junction of circRNAs was validated by Sanger sequencing. The arrow showed the "head-tail" splicing sites of circRNA. 
A

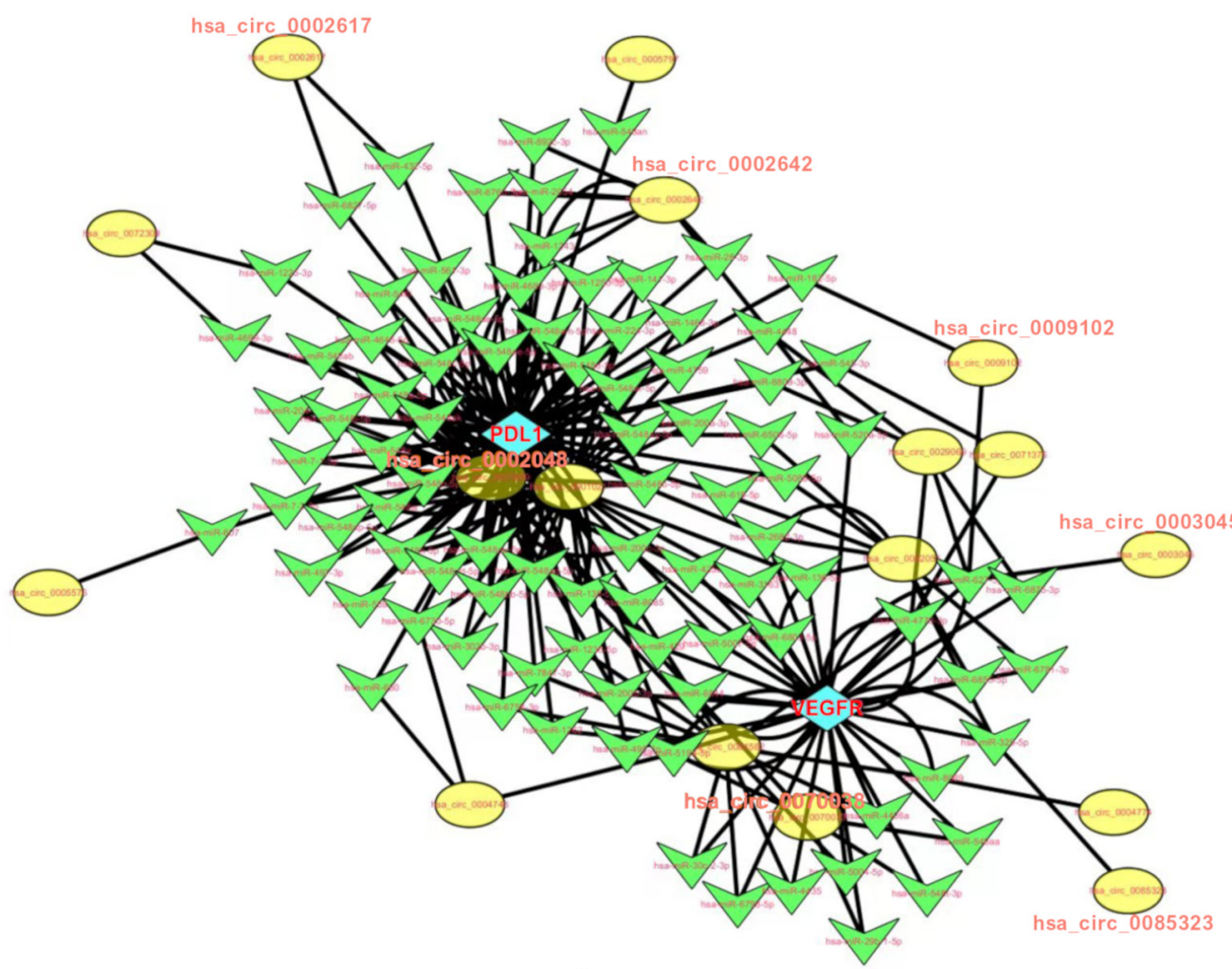

B

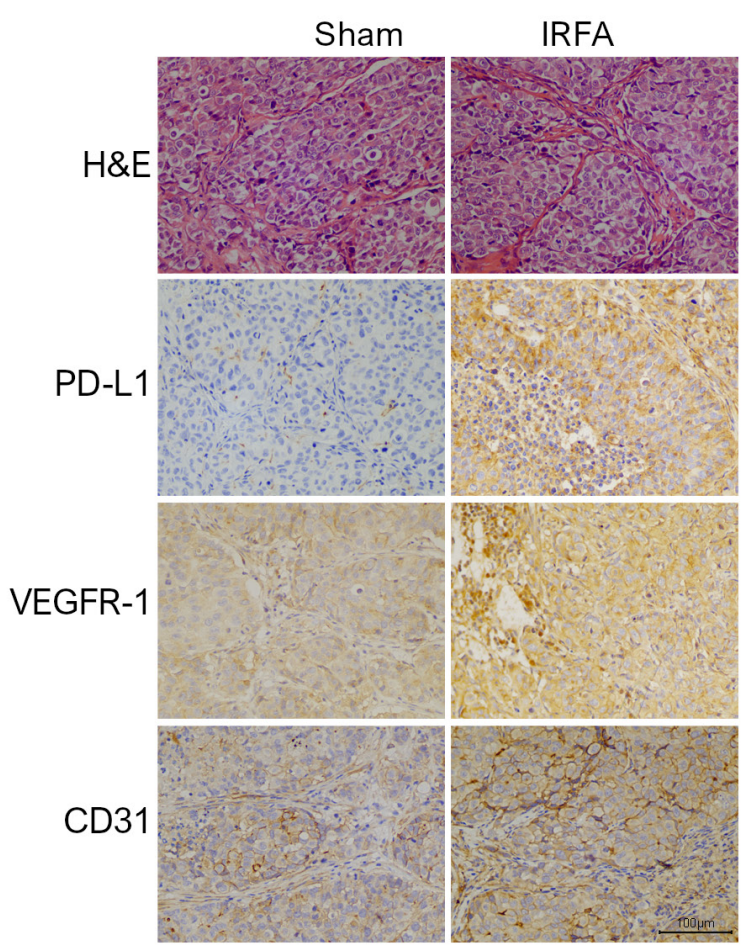

C

Sham
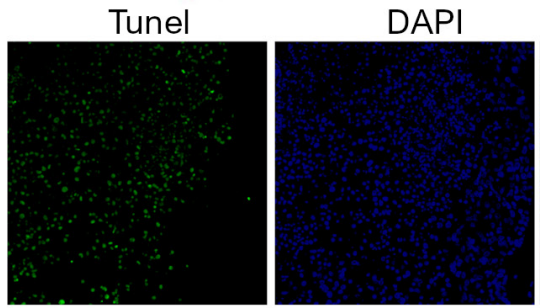

IRFA
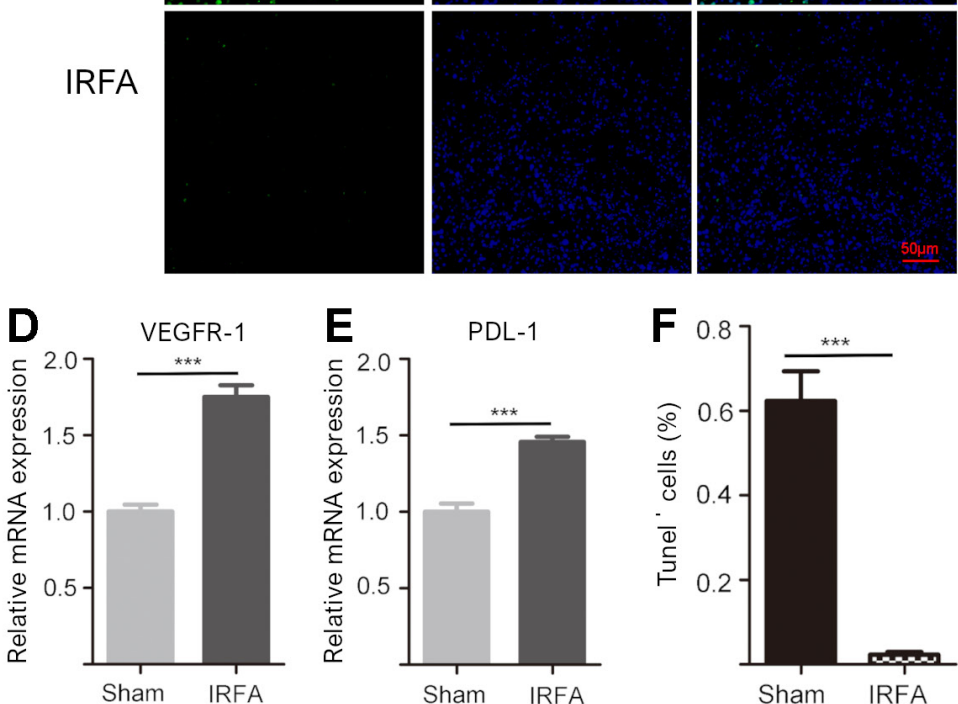

Figure 6. Prediction of circRNAs/miRNAs/PD-L1 and VEGFR-1 network. A) The relationships between circRNAs, miRNAs, and PD-L1/VEGFR-1 were confirmed by the IHC staining (B) for PD-L1, VEGFR-1, and CD31 in xenograft tumor. C, F) Apoptosis was examined by TUNEL assay. D, E) The relative mRNAs expression of PD-L1 and VEGFR-1 detected by qRT-PCR in IRFA-treated tumor tissues and control group. Data were expressed as the mean \pm SD and reproduced in three independent experiments. 


\section{Discussion}

RFA has been widely used for the treatment of small HCCs $(<3 \mathrm{~cm})$ with encouraging results. Surprisingly, local recurrence of HCC was reported to progress rapidly after RFA [4, 5], especially in patients with IRFA, and the causes of this phenomenon remain largely unclear [22]. Several studies revealed that IRFA could promote rapid progression of residual tumors through the hypoxia inducible factor-1a (HIF-1a)/vascular endothelial growth factor (VEGF) pathway and improve the invasion of residual HCC [22]. Fukuda et al. proved that pre-RFA sorafenib administration might decrease blood flow in tumor or non-tumor areas by inhibiting VEGF, which could lead to a larger area of RFA coagulation necrosis [23]. The role of PD-L1 expression has also been evaluated in HCC prognosis and treatment response, suggesting that high expression is associated with worse outcomes. Additionally, PD-L1 was previously validated to be correlated with HCC patients' progression-free survival and overall survival [24].

Recent evidence has shown that circRNAs are a special class of noncoding RNAs that are broadly expressed in human cells and tissues and involved in the development of various human diseases [25]. Further mechanistic studies have verified that circRNAs have many functional roles, including functioning as miRNA 'sponges', regulating gene expression at the transcriptional or posttranscriptional level $[6,25]$, binding and sequestering other RNA binding proteins [26, 27], and encoding proteins with functions distinct from those of their canonical counterparts [28]. Previous studies have shown that circRNAs play a vital role in human cancer [25]. For instance, circHIPK3 has been reported to be an oncogenic factor in bladder cancer and colorectal cancer $[29,30]$, whereas circFAT1 displays inhibitory effects in GC [31], repressing the proliferation and invasion of GC cells. It has been suggested that circRNAs might act as potential diagnostic and prognostic biomarkers in HCC [15]. For example, circSETD3 can serve as a novel tumor suppressor in HCC and is a valuable prognostic biomarker [32], and downregulation of hsa_circ_0001649 in HCC predicts a poor prognosis [33]. Other studies demonstrated that circRNA MTO1 acts as a sponge for miR-9 to suppress the progression of hepatocellular carcinoma [13]. However, the role of circRNAs in HCC after IRFA has rarely been explored.

In this study, tumor progression was enhanced in our mice model. Subsequently, the results of circRNA sequencing identified a total of 612 differentially expressed circRNAs between the IRFA-treated subcutaneous HCC and control groups, among which 435 circRNAs were upregulated and 177 were downregulated. GO and KEGG pathway analyses were used to functionally annotate parental genes of differentially expressed circRNAs. These parental genes were predicted to be functionally related to metabolic pathways, viral carcinogenesis, the Ras signaling pathway, the Wnt signaling pathway, the cAMP signaling pathway, and so on.
Moreover, seven selected circRNAs were overexpressed in the IRFA group by qRT-PCR, and the results were consistent with those of RNA-seq, demonstrating that this circRNA profile is highly reliable. Hence, our study revealed that changes in circRNA expression alterations were closely associated with the progression of HCC in an IRFA model.

Previous studies demonstrated that circRNAs mainly function as miRNA sponges to sequester miRNAs and then regulate subsequent gene expression. Thus, to investigate whether the seven circRNAs might exert effects on VEGFR-1 and PD-L1 mRNA levels by serving as "miRNA sponges", we predicted their potential target miRNAs and constructed circRNA-miRNA regulation networks utilizing bioinformatic analysis. The results indicated that seven circRNAs were predicted to likely regulate PD-L1 and VEGFR-1 expression levels by acting as sponges for microRNAs (miRNAs), forming a circRNA-miRNA-PD-L1/ VEGFR-1 axis. Then, we found that PD-L1 and VEGFR-1 were overexpressed in the IRFA group, as determined by IHC and qRT-PCR, and initially confirmed that circRNAs might influence PD-L1 and VEGFR-1 expression levels, serving as sponges for miRNAs. Although we determined the circRNA profile in the IRFA HCC model and indicated their potential functions and mutual mechanism in HCC by bioinformatics for the first time, the precise underlying molecular regulation of circRNAs in the development and progression of HCC followed by IRFA treatment needs further study. First, as our results revealed a distinguishable circRNA expression profile of the samples harvested from IRFA HCC model mice, dysregulation of circRNAs and their pathophysiological relevance need to be further explored in clinical samples. Second, based on the sponge theory, ceRNAs should have sufficient functional miRNA binding sites. Further validation of the interaction between differentially expressed circRNAs and their target miRNAs might help clarify the underlying mechanisms of IRFA HCC. In addition to the popular sponge theory, other possible mechanisms, such as the translation of differentially expressed circRNAs associated with IRFA HCC to proteins, remain unclear. Further studies are required to uncover the potential roles of circRNAs in IRFA HCC.

In summary, our study identified that circRNA alterations were involved in the development of an IRFA model. Moreover, seven circRNAs might participate in the biological malignancy of HCC after IRFA treatment by adsorbing downstream miRNAs and thereby affecting expression levels of two target genes, PD-L1 and VEGFR-1. Finally, the roles of these circRNAs need to be further explored systematically according to the circRNA-miRNA-PD-L1/VEGFR-1 network, which could improve our understanding of the pathogenic mechanisms in HCC progression subjected to IRFA treatment and help find new molecular targets for the clinical treatment of HCC.

Supplementary information is available in the online version of the paper. 
Acknowledgments: This work was supported by the Science and Technology Planning Project of Guangdong Province (Nos. 2018A030313700 and 2018A030313511), and Guangzhou Planned Project of Science and Technology (202102010028).

\section{References}

[1] BRAY F, FERLAY J, SOERJOMATARAM I, SIEGEL RL, TORRE LA et al. Global cancer statistics 2018: GLOBOCAN estimates of incidence and mortality worldwide for 36 cancers in 185 countries. CA Cancer J Clin 2018; 68: 394-424. https://doi.org/10.3322/caac.21492

[2] LLOVET JM, ZUCMAN-ROSSI J, PIKARSKY E, SANGRO B, SCHWARTZ $M$ et al. Hepatocellular carcinoma. Nat Rev Dis Primers 2016; 2: 16018. https://doi.org/10.1038/ nrdp.2016.18

[3] MARRERO JA, KULIK LM, SIRLIN CB, ZHU AX, FINN RS et al. Diagnosis, Staging, and Management of Hepatocellular Carcinoma: 2018 Practice Guidance by the American Association for the Study of Liver Diseases. Hepatology 2018; 68: 723-750. https://doi.org/10.1002/hep.29913

[4] PORTOLANI N, TIBERIO GA, RONCONI M, CONIGLIO A, GHIDONI $S$ et al. Aggressive recurrence after radiofrequency ablation of liver neoplasms. Hepatogastroenterology 2003; 50: 2179-2184.

[5] RUZZENENTE A, MANZONI GD, MOLFETTA M, PACHERA S, GENCO B et al. Rapid progression of hepatocellular carcinoma after Radiofrequency Ablation. World J Gastroenterol 2004; 10: 1137-1140. https://doi.org/10.3748/ wjg.v10.i8.1137

[6] MEMCZAK S, JENS M, ELEFSINIOTI A, TORTI F, KRUEGER J et al. Circular RNAs are a large class of animal RNAs with regulatory potency. Nature 2013; 495: 333-338. https://doi.org/10.1038/nature11928

[7] SANG M, MENG L, SANG Y, LIU S, DING P et al. Circular RNA ciRS-7 accelerates ESCC progression through acting as a miR-876-5p sponge to enhance MAGE-A family expression. Cancer Lett 2018; 426: 37-46. https://doi.org/10.1016/j. canlet.2018.03.049

[8] LI F, ZHANG L, LI W, DENG J, ZHENG J et al. Circular RNA ITCH has inhibitory effect on ESCC by suppressing the Wnt/ $\beta$-catenin pathway. Oncotarget 2015; 6: 6001-6013. https://doi.org/10.18632/oncotarget.3469

[9] CHEN J, LI Y, ZHENG Q, BAO C, HE J et al. Circular RNA profile identifies circPVT1 as a proliferative factor and prognostic marker in gastric cancer. Cancer Lett 2017; 388: 208219. https://doi.org/10.1016/j.canlet.2016.12.006

[10] ZHANG J, LIU H, HOU L, WANG G, ZHANG R et al. Circular RNA_LARP4 inhibits cell proliferation and invasion of gastric cancer by sponging miR-424-5p and regulating LATS1 expression. Mol Cancer 2017; 16: 151. https://doi. org/10.1186/s12943-017-0719-3

[11] LI X, WANG J, ZHANG C, LIN C, ZHANG J et al. Circular RNA circITGA7 inhibits colorectal cancer growth and metastasis by modulating the Ras pathway and upregulating transcription of its host gene ITGA7. J Pathol 2018; 246: 166-179. https://doi.org/10.1002/path.5125
[12] HSIAO KY, LIN YC, GUPTA SK, CHANG N, YEN L et al. Noncoding Effects of Circular RNA CCDC66 Promote Colon Cancer Growth and Metastasis. Cancer Res 2017; 77: 2339-2350. https://doi.org/10.1158/0008-5472.CAN-161883

[13] HAN D, LI J, WANG H, SU X, HOU J et al. Circular RNA circMTO1 acts as the sponge of microRNA-9 to suppress hepatocellular carcinoma progression. Hepatology 2017; 66: 1151-1164. https://doi.org/10.1002/hep.29270

[14] YU J, XU QG, WANG ZG, YANG Y, ZHANG L et al. Circular RNA cSMARCA5 inhibits growth and metastasis in hepatocellular carcinoma. J Hepatol 2018; 68: 1214-1227. https://doi.org/10.1016/j.jhep.2018.01.012

[15] WANG M, YU F, LI P. Circular RNAs: Characteristics, Function and Clinical Significance in Hepatocellular Carcinoma. Cancers (Basel) 2018; 10: 258 https://doi.org/10.3390/cancers 10080258

[16] ASHWAL-FLUSS R, MEYER M, PAMUDURTI NR, IVANOV A, BARTOK O et al. circRNA biogenesis competes with pre-mRNA splicing. Mol Cell 2014; 56: 55-66. https:// doi.org/10.1016/j.molcel.2014.08.019

[17] HANSEN TB, JENSEN TI, CLAUSEN BH, BRAMSEN JB, FINSEN B et al. Natural RNA circles function as efficient microRNA sponges. Nature 2013; 495: 384-388. https://doi. org/10.1038/nature11993

[18] YE G, HUANG K, YU J, ZHAO L, ZHU X et al. MicroRNA-647 Targets SRF-MYH9 Axis to Suppress Invasion and Metastasis of Gastric Cancer. Theranostics 2017; 7: 33383353. https://doi.org/10.7150/thno.20512

[19] LI X, YANG L, CHEN LL. The Biogenesis, Functions, and Challenges of Circular RNAs. Mol Cell 2018; 71: 428-442. https://doi.org/10.1016/j.molcel.2018.06.034

[20] BACHMAYR-HEYDA A, REINER AT, AUER K, SUKHBAATAR N, AUST $S$ et al. Correlation of circular RNA abundance with proliferation--exemplified with colorectal and ovarian cancer, idiopathic lung fibrosis, and normal human tissues. Sci Rep 2015; 5: 8057. https://doi.org/10.1038/ srep08057

[21] SALZMAN J, CHEN RE, OLSEN MN, WANG PL, BROWN PO. Cell-type specific features of circular RNA expression. Plos Genet 2013; 9: e1003777. https://doi.org/10.1371/journal.pgen. 1003777

[22] KONG J, KONG J, PAN B, KE S, DONG S et al. Insufficient radiofrequency ablation promotes angiogenesis of residual hepatocellular carcinoma via HIF-1 $\alpha /$ VEGFA. Plos One 2012; 7: e37266. https://doi.org/10.1371/journal. pone.0037266

[23] FUKUDA H, NUMATA K, MORIYA S, SHIMOYAMA Y, ISHII $\mathrm{T}$ et al. Hepatocellular carcinoma: concomitant sorafenib promotes necrosis after radiofrequency ablation-propensity score matching analysis. Radiology 2014; 272: 598-604. https://doi.org/10.1148/radiol.14131640

[24] MOCAN T, ILIES M, NENU I, CRACIUN R, HORHAT A et al. Serum levels of soluble programmed death-ligand 1 (sPD-L1): A possible biomarker in predicting post-treatment outcomes in patients with early hepatocellular carcinoma. Int Immunopharmacol 2021; 94: 107467. https://doi. org/10.1016/j.intimp.2021.107467 
[25] LI X, YANG L, CHEN LL. The Biogenesis, Functions, and Challenges of Circular RNAs. Mol Cell 2018; 71: 428-442. https://doi.org/10.1016/j.molcel.2018.06.034

[26] HUANG X, HE M, HUANG S, LIN R, ZHAN M et al. Circular RNA circERBB2 promotes gallbladder cancer progression by regulating PA2G4-dependent rDNA transcription. Mol Cancer 2019; 18: 166. https://doi.org/10.1186/s12943019-1098-8

[27] YANG Q, DU WW, WU N, YANG W, AWAN FM et al. A circular RNA promotes tumorigenesis by inducing c-myc nuclear translocation. Cell Death Differ 2017; 24: 1609-1620. https://doi.org/10.1038/cdd.2017.86

[28] LEGNINI I, DI TIMOTEO G, ROSSI F, MORLANDO M, BRIGANTI $\mathrm{F}$ et al. Circ-ZNF609 Is a Circular RNA that Can Be Translated and Functions in Myogenesis. Mol Cell 2017; 66: 22-37. https://doi.org/10.1016/j.molcel.2017.02.017

[29] LI Y, ZHENG F, XIAO X, XIE F, TAO D et al. CircHIPK3 sponges miR-558 to suppress heparanase expression in bladder cancer cells. Embo Rep 2017; 18: 1646-1659. https://doi. org/10.15252/embr.201643581
[30] ZENG K, CHEN X, XU M, LIU X, HU X et al. CircHIPK3 promotes colorectal cancer growth and metastasis by sponging miR-7. Cell Death Dis 2018; 9: 417. https://doi. org/10.1038/s41419-018-0454-8

[31] FANG J, HONG H, XUE X, ZHU X, JIANG L et al. A novel circular RNA, circFAT1(e2), inhibits gastric cancer progression by targeting miR-548g in the cytoplasm and interacting with YBX1 in the nucleus. Cancer Lett 2019; 442: 222-232. https://doi.org/10.1016/j.canlet.2018.10.040

[32] XU L, FENG X, HAO X, WANG P, ZHANG Y et al. CircSETD3 (Hsa_circ_0000567) acts as a sponge for microRNA-421 inhibiting hepatocellular carcinoma growth. J Exp Clin Cancer Res 2019; 38: 98. https://doi.org/10.1186/ s13046-019-1041-2

[33] ZHANG X, QIU S, LUO P, ZHOU H, JING W et al. Downregulation of hsa_circ_0001649 in hepatocellular carcinoma predicts a poor prognosis. Cancer Biomark 2018; 22: 135142. https://doi.org/10.3233/CBM-171109 
https://doi.org/10.4149/neo_2021_210817N1170

\section{Identification of circRNAs involved in the development of hepatocellular carcinoma after insufficient radiofrequency ablation}

Jun-Wei CHEN ${ }^{1, *}$, Li-Sha LAI ${ }^{2, *}$, Jun-Yang LUO ${ }^{1}$, Chu-Ren ZHOU ${ }^{1}$, Min-Gan LI' ${ }^{1}$, Ming-Sheng HUANG ${ }^{1, *}$, Ping WU ${ }^{3, *}$

\section{Supplementary Information}

\begin{tabular}{|c|c|c|}
\hline List of oligonucleotide sequences & 5' to 3' & Length (bp) \\
\hline hsa_circ_0029069-CF & TGAAGAAACTTAGACAGCAGCT & \multirow{2}{*}{170} \\
\hline hsa_circ_0029069-CR & TCTTCGGAGCTCAGCTACTT & \\
\hline hsa_circ_0029069-LF & TGGAGCATGCCAACAAAGAG & \multirow{2}{*}{144} \\
\hline hsa_circ_0029069-LR & CAAATTCTGCCGTCTCTGTTCC & \\
\hline hsa_circ_0027689-CF & СTTCTTACAGGCTACCCATGTTC & \multirow{2}{*}{117} \\
\hline hsa_circ_0027689-CR & ATAGCAGGCAGTAACCACACC & \\
\hline hsa_circ_0027689-LF & TGGACTCCAATTGCCTTGAC & \multirow{2}{*}{115} \\
\hline hsa_circ_0027689-LR & ACCCCTGGGCAATAAACACTTC & \\
\hline hsa_circ_0003288-CF1 & AGGTTGGTCCTACAGCTACAC & \multirow{2}{*}{143} \\
\hline hsa_circ_0003288-CR1 & TGCGTGCAAGAACCTTTCAG & \\
\hline hsa_circ_0003288-LF & CAAGCAGCAAACCAAACCAG & \multirow{2}{*}{142} \\
\hline hsa_circ_0003288-LR & TCAGTGTGAGCCACAATTGG & \\
\hline hsa_circ_0001021-CF & AGCAACTTCCAAAGGAGCAG & \multirow{2}{*}{113} \\
\hline hsa_circ_0001021-CR & CGAATGATGTCTGGCTCCATTG & \\
\hline hsa_circ_0001021-LF & TGGCTTTGGGTAGAAGCTTG & \multirow{2}{*}{136} \\
\hline hsa_circ_0001021-LR & TCATCCTGTTGCAGTGTTGG & \\
\hline hsa_circ_0084582-CF & ACCAGTCTTCAССТССАСАС & \multirow{2}{*}{137} \\
\hline hsa_circ_0084582-CR & ATTCTCTTGGACCCTGCAGTG & \\
\hline hsa_circ_0084582-LF & ACAACTCAGGGCAGTATTCTCG & \multirow{2}{*}{150} \\
\hline hsa_circ_0084582-LR & ATGGGAATCCTACAGCATTGGG & \\
\hline Hsa-GAPDH-F & GAGTCAACGGATTTGGTCGT & \multirow{2}{*}{185} \\
\hline Hsa-GAPDH-R & GACAAGCTTCCCGTTCTCAG & \\
\hline Hsa-GAPDH-circ-F & TCTGACTTCAACAGCGACAC & \multirow{2}{*}{214} \\
\hline Hsa-GAPDH-circ-R & TGACGGTGCCATGGAATTTG & \\
\hline
\end{tabular}

